\title{
Investigation on Structural and Optical properties of Cadmium doped Lead Oxide Nanoparticles
}

\author{
S. G. Rejith ${ }^{1}$, G. Sudha ${ }^{2}$ \\ Department of Physics, St. Xavier's College, Palayamkottai, India 1,2
}

\begin{abstract}
Cd-doped $\mathrm{PbO}$ is an inorganic metal oxide nanoparticle, was successfully synthesized by a simple microwave assisted solvothermal method. The prepared sample was characterized by using Powder X-Ray Diffraction (PXRD), Scanning Electron Microscopy (SEM), Fourier Transform Infrared Spectroscopy (FTIR), UVVisible spectroscopy (UV) and Photoluminescence (PL) spectroscopy. Using PXRD pattern, the average crystallite size was calculated as $74.09 \mathrm{~nm}$. The morphology of the prepared Cd-doped $\mathrm{PbO}$ powdered sample was analyzed by the SEM micrograph. FTIR is a technique used to measure the vibrational frequencies of bonds in the molecule. Band gap of the $\mathrm{Cd}$-doped $\mathrm{PbO}$ nanoparticle found using Tauc plots. PL emission spectrum of $\mathrm{Cd}$-doped $\mathrm{PbO}$ nanoparticle was investigated. The emission spectrum revealed a blue emission.
\end{abstract}

Keywords: Nanoparticles, Powder technology, Structural, Optical materials and properties.

\section{INTRODUCTION}

Among the various classes of inorganic nanoparticles, metal oxide nanoparticles are particularly attractive from both the scientific and technological point of view [1-3]. The metal elements are able to form a large diversity of oxide compounds [4]. When metal oxides are brought into the nanometer scale, they further exhibit improved or completely novel properties compared to their bulk materials [5-9]. Recently considerable research efforts have been directed toward the preparation of binary oxides with 1D nanostructure.

Lead (II) oxide (PbO), is a member of the relatively small family of lead(II) binary oxides, which also includes $\mathrm{PbO}_{2}$ and $\mathrm{Pb}_{3} \mathrm{O}_{4}$. Both $\mathrm{PbO}$ and $\mathrm{PbO}_{2}$ are semiconducting metal oxides [10]. PbO occurs in two polymorphs, one having a tetragonal crystal structure and the other having an orthorhombic crystal structure. As determined by X-ray crystallography, both polymorphs, tetragonal and orthorhombic feature a pyramidal four-coordinate $\mathrm{Pb}$ center.The pyramidal nature indicates the presence of a stereo-chemically active lone pair of electrons [11]. When $\mathrm{PbO}$ occurs in tetragonal lattice structure it is called litharge and when the $\mathrm{PbO}$ has orthorhombic lattice structure it is called massicot[12]. The tetragonal form is usually red or orange color, while the orthorhombic is usually yellow or orange, but the color is not a very reliable indicator of the structure [13]. Lead oxide is widely used in different industries such as network modifiers in luminescent glassy materials, pigments, gas sensors, paints, lead-acid batteries and nanoscale electronic devices [14-20].

There are several methods have been developed to synthesize the lead oxide nanoparticles. Among them, solvothermal process has been shown to be a powerful technique for generating novel materials with interesting properties. The present work is focused on the preparation of $\mathrm{Cd}$-doped $\mathrm{PbO}$ nanoparticles by microwave assisted solvothermal method.

\section{MATERIALS AND METHODS}

$\mathrm{Cd}$-doped $\mathrm{PbO}$ nanoparticle was successfully prepared by a simple microwave assisted solvothermal method. In order to synthesize the Cd-doped PbO Nanoparticles, the Analytical Reagent (AR) grade Lead acetate trihydrate $\left(\mathrm{Pb}\left(\mathrm{CH}_{3} \mathrm{COO}\right)_{2} \cdot 3 \mathrm{H}_{2} \mathrm{O}\right)$, Urea $\left(\mathrm{CH}_{4} \mathrm{~N}_{2} \mathrm{O}\right)$ and Ethylene glycol $\left(\mathrm{CH}_{2}(\mathrm{OH}): \mathrm{CH}_{2} \mathrm{OH}\right)$ (as solvent) are used as initial precursors. Lead acetate trihydrate and urea are taken as solute in the molecular ratio 1:3 and dissolved in $100 \mathrm{ml}$ ethylene glycol as individually.

For Cadmium doping, cadmium acetate dihydrate $(\mathrm{Cd}$ $\left.\left(\mathrm{CH}_{3} \mathrm{COO}\right)_{2} \cdot 3 \mathrm{H}_{2} \mathrm{O}\right)(5 \mathrm{wt} \%)$ are added with the above precursors. Then the solutions are stirred well separately by using magnetic stirrer, a clear solution was obtained, the prepared solutions are mixed together and kept in a microwave oven (operated with frequency $2450 \mathrm{MHz}$ and power $700 \mathrm{~W}$ ) for 40 minutes.

The precipitate will obtain, when the solvent is evaporated completely. The obtained precipitate is washed with distilled water and acetone $\left(\mathrm{CH}_{3} \mathrm{COCH}_{3}\right)$ several times to remove the organic impurities. Then the sample was dried in atmospheric air and collected the yield.

\section{RESULT AND DISCUSSION}

The current work is devoted to the investigation of $\mathrm{Cd}$ doped $\mathrm{PbO}$ nanoparticles formation from metal-organic compound, which have been prepared by using simple microwave assisted solvothermal method, was characterized using PXRD, SEM, FTIR, UV-Vis and PL is given below: 


\subsection{PXRD Analysis}

The crystalline structure of the sample was investigated by $\mathrm{X}$-Ray powder diffractometer operating with $\mathrm{CuK}$ wavelength $1.54056 \AA$ and the data was taken for the diffraction angle ranging from $20^{\circ}$ to $70^{\circ}$.

Fig.1, shows the PXRD patterns of $\mathrm{Cd}$ - doped $\mathrm{PbO}$ nanoparticles. The PXRD patterns are compared well with the available literature which indicates the sample prepared in the present study is basically $\mathrm{PbO}$ nanoparticles. The diffraction peaks were indexed to the orthorhombic system of $\mathrm{PbO}$ with lattice parameter $\mathrm{a}=4.971 \AA, \mathrm{b}=5.956 \AA, \mathrm{c}=5.438 \AA$ which are in agreement with the reported values [JCPDS(Joint Committee on Powder Diffraction Standards) 88-1589]. No characteristics peaks of $\mathrm{Cd}$ have been observed that means dopant do not affect the crystal structure.The crystallite size calculated by Debye-Scherer formula,

$\mathrm{D}=\mathrm{k} \lambda / \beta \cos \theta$

The average crystallite size of the Cd-doped $\mathrm{PbO}$ crystallite calculated to be about 74.09

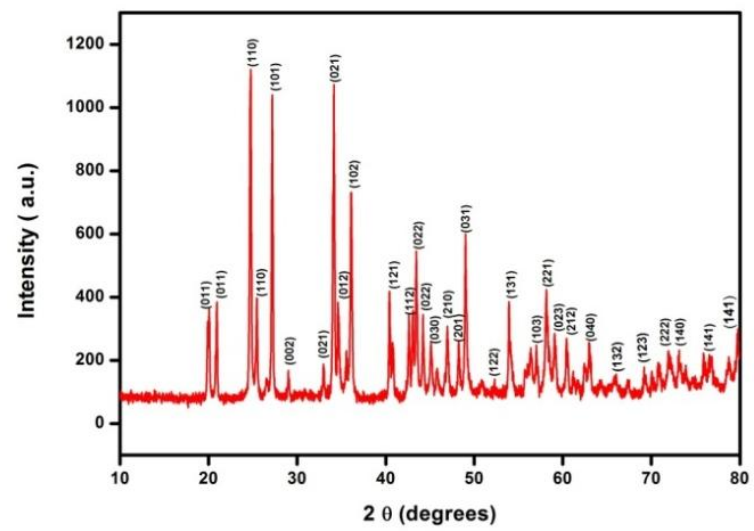

Fig. 1 PXRD pattern of Cd-doped PbO Nanoparticles

\subsection{SEM Analysis}

Morphology and structure of sample was further investigated by SEM analysis. The SEM micrograph of The band gap spectra of Cd-doped PbO nanocrystal the $\mathrm{Cd}$-doped $\mathrm{PbO}$ nanoparticle are shown in the fig. 2 . obtained in the present study is presented in fig. 4 . A graph SEM photograph clearly show the surface features, which indicates that the nanoparticle was successfully prepared.

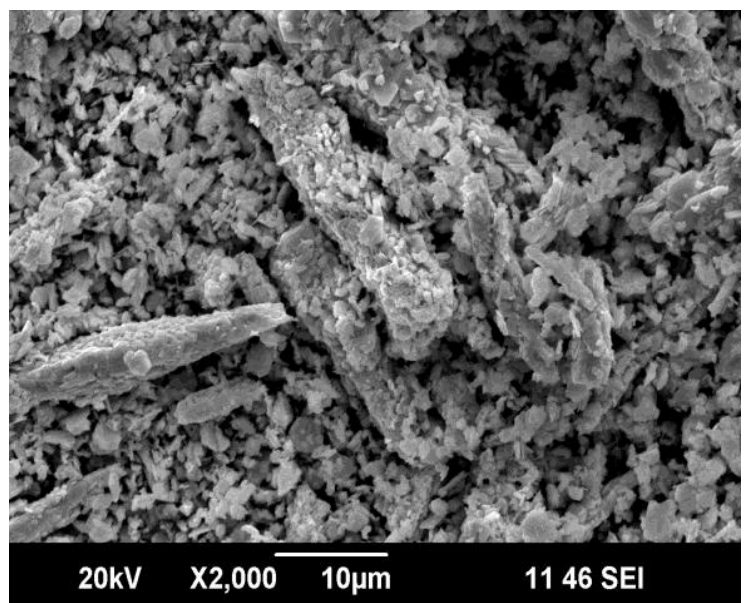

Fig. 2 SEM Micrographs of Cd-doped PbO nanoparticles.
The instrumental parameters, accelerating voltage, spot size, and magnification and working distances are indicated on SEM image. The Cd-doped $\mathrm{PbO}$ nanoparticle are having rocky stone like morphology, it can be seen that the particles congregate together. The average diameter of the particle observed from SEM analysis is $200 \mathrm{~nm}$, which is larger than the diameter predicted from X-Ray broadening.

\subsection{FTIR Analysis}

Fig. 3, shows the FTIR spectrum of the Cd-doped $\mathrm{PbO}$ nanoparticle, which was acquired in the range of 400-4000 $\mathrm{cm}^{-1}$.The peak at the $3463 \mathrm{~cm}^{-1}$ belongs to the $-\mathrm{OH}$ stretching vibration, the peak $1639 \mathrm{~cm}^{-1}$ attributed to the $\mathrm{H}-\mathrm{O}-\mathrm{H}$ bond bending vibrations. These vibrations arise due to the sample absorb moisture from the surroundings during the sample preparation. The bond at $680 \mathrm{~cm}^{-1} \& 416 \mathrm{~cm}^{-1}$ is assigned to $\mathrm{Pb}-\mathrm{O}$ bond vibrations. It confirmed that the final product is the presence of lead and oxide.

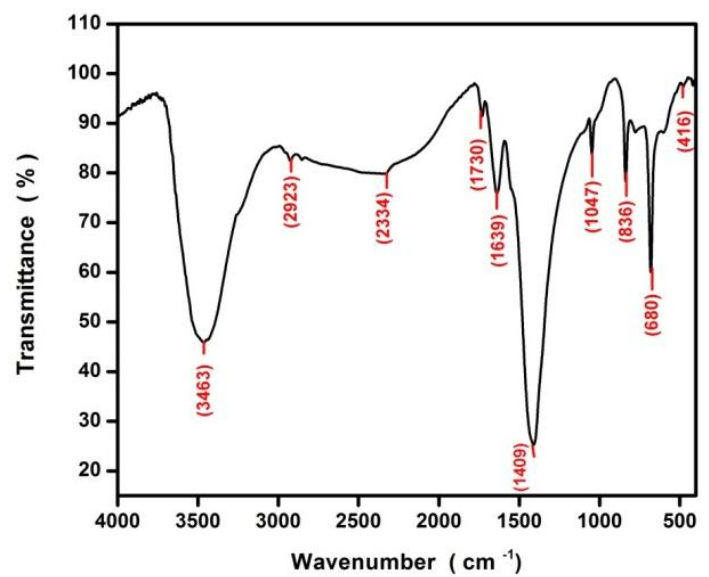

Fig.3 FTIR spectrum for Cd-doped PbO Nanoparticles. of $\left((\alpha h v)^{2}\right.$ versus $\left.h v\right)$ is as shown in Figure, by extrapolating the graph to $\mathrm{X}$ axis in order to calculated the band gap of the sample. The band gap is found to be 4.61 $\mathrm{eV}$. The observed band gap is nearly good agreement with the expected value.

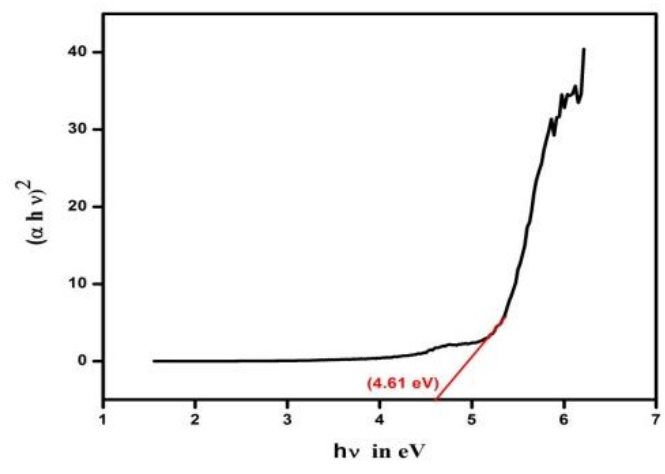

Fig.4 Band gap spectra for Cd-doped PbO Nanoparticles. 


\subsection{PL Analysis}

Fig. 5, shows the PL emission spectrum of Cd-doped $\mathrm{PbO}$ nanoparticle. The emission spectrum revealed nearly a blue emission, which might be related with oxygen vacancies. Thus, the blue emission could also be attributed to recombination of electrons in the conduction band with deep doubly ionized oxygen vacancies.

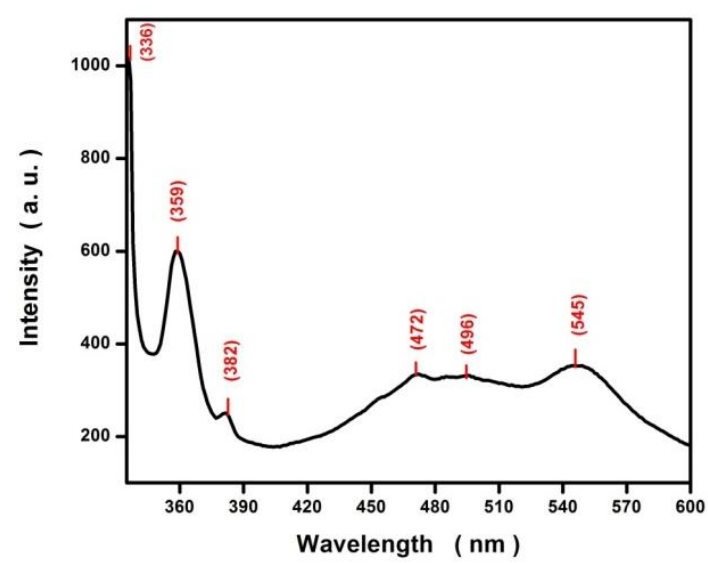

Fig.5 PL emission spectra for Cd-doped $\mathrm{PbO}$ Nanoparticles.

\section{CONCLUSION}

Cd-doped $\mathrm{PbO}$ nanoparticle has been synthesized successfully by microwave assisted solvothermal method.From the PXRD spectrum, the average size of the prepared nanoparticle was found to be $74.09 \mathrm{~nm}$. SEM micrographs clearly show the surface features, by which points that the $\mathrm{Cd}$-doped $\mathrm{PbO}$ nanoparticle was successfully prepared.The FTIR spectrum of the samples were recorded in the range $4000-400 \mathrm{~cm}^{-1}$, which confirmed that the final product is the presence of lead, cadmium and oxide.From the UV-Visible spectroscopy, the band gap is found to be $4.61 \mathrm{eV}$. From the PL emission spectrum, the emission spectrum, the emission band revealed a blue emission.

\section{REFERENCES}

[1] Noguera, C. "Physics and Chemistry at Oxide Surfaces", Cambridge: 1996.

[2] Kung, H.H. "Transition Metal Oxides: Surface Chemistry and Catalysis", $6^{\text {th }}$ ed. Amsterdam, the Netherlands: 1989.

[3] Henrich, V.E.; Cox, P.A. "The Surface Chemistry of Metal Oxides", Cambridge: 1994.

[4] Wyckoff, R.W.G. "Crystal Structures", New York: 1964.

[5] Gleiter, H. Nanostruct. J. Mater. 1995, 6, 3.

[6] Valden, M.; Lai, X.; Goodman, D.W. Science, 1998, 281, 1647.

[7] Rodriguez, J.A.; Liu, G.; Jirsak, T.; Hrbek, Chang, Z.; Dvorak, J.; Maiti, A. J. Am. Chem. Soc. 2002, 124, 5247.

[8] Baumer, M.; Freund, H.-J. Progress in Surf.Sci. 1999, 61, 127.

[9] Trudeau, M.L.; Ying, J.Y Nanostruct. Mater.1996, 7, 245.

[10] P. Mattesco, N. Bui, P. Simon, L. Albert: J. Power Sources 64, 21 (1997).

[11] Wells, A. F., "Structural Inorganic Chemistry", 5th ed. Clarendon : 1984

[12] Anil Kumar De “A Text Book of Inorganic Chemistry”, New Age International: 2007.
[13] David John Rowe, "Lead manufacturing in Britain", London: 1983.

[14] Thulasiramudu, S. Buddhudu, Spectrochim. Acta A 66 (2007) 323.

[15] T.L. Blair, J. Power Sources 73 (1998) 47. 16 International Journal of NanomaterialsandBiostructures 2011; 1 (2) 12-16

[16] P. Veluchamy, M. Sharon, M. Shimizu, H. Minoura, J. Electroanal. Chem. 365 (1994) 179.

[17] S. Ghasemi, M.F. Mousavi, M. Shamsipur, H. Karami, Ultrason.Sonochem.15(2008) 448

[18] C. Barriga, S. Maffi, L.P. Bicelli, C. Malitesta, J. Power Sources 34 (1991) 353.

[19] W.U. Huynh, J.J. Dittmer, A.P. Alivisatos, Science 295 (2002) 2425.

[20] G. Xi, Y. Peng, L. Xu, M. Zhang, W. Yu, Y. Qian, Inorg. Chem. Commun.7 (2004) 607.

\section{BIOGRAPHY}

S. G. Rejith, Professor in physics, St. Xaviers College, Palayamkottai. 